
\title{
3 Research Square \\ The influenza vaccine for nursing and care professionals at emergency services in Flanders
}

Matthijs Samyn ( $\nabla$ matthijs.samyn@sintandriestielt.be )

Sint-Andries hospital https://orcid.org/0000-0002-5716-1695

Guido Van Hal

Universiteit Antwerpen

Herman Vandevijvere

Karel de Grote-Hogeschool Antwerpen

Pierre Van Damme

Universiteit Antwerpen

Research article

Keywords: Vaccination, flu, absenteeism, nursing and care professionals, emergency services, Flanders

Posted Date: July 23rd, 2020

DOI: https://doi.org/10.21203/rs.3.rs-42512/v1

License: (c) (i) This work is licensed under a Creative Commons Attribution 4.0 International License.

Read Full License

Version of Record: A version of this preprint was published at Human Vaccines \& Immunotherapeutics on April 12th, 2021. See the published version at https://doi.org/10.1080/21645515.2021.1894062. 


\section{Abstract}

Background: The Belgian Superior Health Council recommends seasonal influenza vaccination for people working in the health care sector in order to reduce the risk of the influenza virus being transmitted to people at risk. The aim of this study is to provide more insight into the current vaccination rate in nurses and health care professionals in emergency services in Flanders. In addition, the influence of influenza vaccination on absenteeism will be investigated, as well as the motivation to get vaccinated or not.

Methods: A quantitative multicentre, cross-sectional study was carried out in which nurses and health care professionals in emergency services in Flanders were questioned by means of an online questionnaire.

Results: The survey of nurses and health care professionals in Flanders from 2018 shows that $54 \%$ $(294 / 548)$ gets vaccinated annually. Respondents who have been vaccinated report a lower absenteeism due to flu compared to health workers who do not get vaccinated. There is a lack of general knowledge about flu and vaccination. $34 \%$ of nurses and health care professionals in emergencies do not know the correct definition of flu. The main reasons for being vaccinated are: to protect oneself $(27 \%)$, family members $(21.5 \%)$ and patients (16\%). Reasons for not getting vaccinated are: never having had the flu before (30\%), believing you get the flu because of the vaccination $(12 \%)$, no belief or trust in the vaccine $(19 \%)$.

Conclusions: This study shows that influenza vaccination campaigns should put the emphasis on knowledge. Nursing and care professionals should be convinced of the effective action of the vaccine.

This can increase the vaccination rate for seasonal influenza and as a consequence reduce absenteeism.

\section{Highlights}

- $46 \%$ of nurses and health care professionals in Flanders does not get vaccinated against influenza (2018)

- Getting a flu vaccine is associated with lower absenteeism due to influenza

- The main motives for getting vaccinated against influenza are to protect themselves, their families and patients.

- Misconceptions and lack of confidence in the vaccine are reasons not to vaccinate.

- Knowledge about flu and vaccination is lacking with these health workers

\section{Background}


The World Health Organization (WHO) defines influenza as follows: "Influenza is a viral infection that mainly affects the nose, throat, bronchi and sometimes the lungs. This infection usually lasts about a week and is characterized by a sudden high fever, muscle pain, headache, severe malaise, non-productive cough, sore throat and rhinitis" [1].

Healthcare workers are much more likely to come into contact and be infected with the flu virus than the average healthy adult [2]. This chance obviously depends on the severity of the epidemic and the department where health workers work. Nurses and health care professionals working in a clinical environment are more likely to be infected than their colleagues in a non-clinical environment. In a clinical environment, the risk of infection is greatest in the emergency department, because nurses and health care professionals are more likely to come into contact with patients who have not received a definitive diagnosis. An estimated 5.8 times a year a health worker in a nursing home is exposed to the flu virus. Although not every exposure leads to an effective infection, it is estimated that one in four health workers could be infected during a mild influenza season. This is much higher than the $10 \%$ in the general population [3].

Influenza vaccination reduces the risk of infection and protects the health care worker himself, his colleagues and patients [4]. If the immunity of the health workers is higher, this will have a positive influence on his/her health and will likely reduce the number of patients infected. From this point of view, vaccination is recommended for all healthcare workers $[2,4]$.

To this end, the Flemish vaccination target sets a vaccination rate of at least $80 \%$ for all hospital personnel [5].

In medical literature, there is little to be found about the level of vaccination and its influence on absenteeism among nurses and health care professionals in emergency services in Flanders. Little is also known about the reasons for whether or not to get vaccinated. The aim of this study is to gain more insight into this.

\section{Methods}

A quantitative multicentre, cross-sectional study was carried out in which nurses and health care professionals in emergency services in Flanders were questioned by means of an online questionnaire. Nurses and health care professionals with at least two years of work experience were included.

The questionnaire was drawn up via the Qualtrics ${ }^{\circledR}$ programme and consists of five blocks: demographic data, reasons for whether or not to get vaccinated against influenza, general knowledge about influenza and its campaign, vaccination status and absenteeism due to flu-like symptoms of the last two years. The latter was questioned on the basis of the WHO definition of influenza. As many closed-ended questions as possible were used with different answer options. The questionnaire was based on information needed to answer the research questions. The results from similar studies were also taken into account $[1,4,6,7]$. 
Respondents were followed up on the basis of the number of response forms received. The aim was to achieve a response rate of at least $35 \%$ per participating emergency department. If the response rate was too low, the questionnaire was once again distributed to the target group. Data analysis was carried out using the Statistical Package for the Social Sciences (SPSS) version 23 programme. Using descriptive analyses, the database was checked for missing values, double response forms of the same respondent and errors. Only fully completed response forms were retained for further statistical analysis. A significance level of $p<0.05$ was used.

\section{Results}

After completion of the online survey, 739 participants were registered, the expected response rate was 300 participants. After checking the demographic data for inclusion and exclusion criteria, 548 response forms were selected. The research population is shown in Table 1.

Table 1

Research population and demographic data

\begin{tabular}{|ll|}
\hline Research population and demographic data & \\
\hline $\mathbf{N}=\mathbf{5 4 8}$ & $\%$ \\
\hline Female & $61 \%$ \\
\hline Age category & \\
\hline $20-35$ & $37 \%$ \\
$36-50$ & $39 \%$ \\
$51-65$ & $24 \%$ \\
\hline Educational level & \\
\hline Bachelor of nursing with special professional title 'emergency and intensive' & $85 \%$ \\
\hline Others & $15 \%$ \\
\hline Jobtime & $62 \%$ \\
\hline Full time & \\
\hline Mean (SD) & $19 \mathrm{y}(11)$ \\
\hline Years active as a health worker & \\
\hline
\end{tabular}

\section{Vaccination status}

For the influenza season $2017-2018,54 \%(n=294)$ of the nurses and health care professionals at emergency services in Flanders have been vaccinated. There is no association between vaccination 
status and age. Moreover, no statistical significance can be observed between education level and vaccination. The fact that information about influenza and its consequences is provided by the employer or not was not significantly different for vaccinated versus non-vaccinated employees. The knowledge of the Flemish government's "Protect Yvette" influenza campaign also did not result in a significant difference. These influenza vaccination campaigns in health care institutions primarily aim to avoid the transmission of the influenza virus from the health care workers to the patient. The emphasis is on both knowledge and practical organisation of a vaccination campaign.

\section{General knowledge of influenza}

The WHO definition of influenza is known by $66 \%(n=360)$ of the respondents. $85 \%(n=471)$ of the respondents know that they can infect others with flu during the incubation period. $12 \%(n=65)$ of health workers do not know that they are not allowed to go to work in case of fever and coughing and would therefore continue to work with the symptoms of flu. When asked whether pregnant women should be given the flu vaccine, $75 \%(n=412)$ answered positively and in this way correctly.

Of the 548 respondents, $27 \%(n=147)$ answered the four knowledge questions above correctly. The questions and percentages for the different answer categories are shown again in Table 2. 
Table 2

General knowledge of influenza

$N=548$

n $\%$

1) According to the WHO, influenza is defined as

Correct definition known

(Infection, lasts about a week, and is characterized by sudden high fever, muscle

soreness, headache, severe malaise, non-productive cough, sore throat and rhinitis)

Definition partly known

(Infection, lasts more than two weeks, and is characterized by sudden high fever, muscle soreness, headache, severe malaise, non-productive cough, sore throat and rhinitis)

Definition not known

(Infection, lasts about a week, and is characterized by only high fever and muscle pain)

Total

2) Can I infect others with flu during the incubation period?

Correct answer known "Yes"

Correct answer not known "No"

$\begin{array}{ll}38 & 7\end{array}$

49

8

Total

3) With the symptoms of flu, would I continue to work?

Correct answer not known "Yes"

$65 \quad 12$

Correct answer known "No"

$317 \quad 58$

166

30

Total

548

4) Can a pregnant employee get the flu vaccine?

Correct answer not known "Yes"

412

75

Correct answer known "No"

48

88

16

Total

548 100

\section{Absenteeism}


There is a statistically significant difference $(p<0.001)$ between the vaccinated $(54 \%)$ and nonvaccinated $(46 \%)$ staff with respect to absenteeism. $3 \%(n=10)$ of the respondents who received the flu vaccine were absent due to flu in the last two years. Among the respondents who were not vaccinated, the absence due to influenza was higher, at $11 \%(n=28)$.

\section{Reasons for whether or not to get vaccinated}

The five main reasons for getting vaccinated are: not having to go through the flu yourself $(27 \%)$, to protect family members $(21.5 \%)$, to protect the patient $(16 \%)$, obliged by the employer $(14 \%)$ and social pressure $(7 \%)$.

The main reasons among the respondents for not getting vaccinated were "Never had a flu" 30\%, "No specific reasons" (24\%), "No faith or confidence in the vaccine" (19\%) and "I get a flu from this vaccination" with $12 \%$.

\section{Discussion}

The results of this study are in line with the results of the Flemish Community study, which shows that only 1 in 2 of all staff in hospitals are vaccinated against influenza every year [5]. An approach to increase the vaccination status is necessary.

The knowledge about how and why getting vaccinated is a strong influence factor for whether or not to be vaccinated [8]. Therefore it is opportune for nursing and care professionals in the emergency department to focus on a better knowledge of the causes and consequences of influenza. The effectiveness of influenza vaccination in high-risk individuals should also be emphasised in future influenza vaccination campaigns $[8,9]$. It is remarkable that $61 \%$ of non-vaccinated people were sufficiently informed. These figures show that responding to the reasons for vaccinating - protecting oneself, family members and patients - can be an added value. On top of that, it will be important in the campaign to focus on the credibility of the vaccine. No belief or trust in the vaccine is one of the biggest factors for not being vaccinated [11].

According to Pereira et al. (2017), a 10\% increase in the number of vaccinees would reduce absenteeism by $10 \%$ [10]. Raising the vaccination status among Flemish nurses and health care professionals in emergency departments could therefore probably also contribute to lower absenteeism. As a result, the spread of flu will also decrease among colleagues and family, and absenteeism may decrease even further. It is important to note here that absenteeism due to influenza has been determined by a selfquestioning of the health care worker. A more objective investigation into the absence of flu related to the data from the human resources department could provide even more clarity.

\section{Conclusions}

The results of this research show that nurses and health care professionals can strongly contribute to the fight against influenza inside and outside the hospital by being vaccinated. These results should be 
interpreted with caution because of the self-reporting of absenteeism. In addition, the response rate should give rise to caution in this conclusion. As not all participating hospitals have replied, a bias may arise. In addition, we would like to underline the importance of preventing and minimising the spread of influenza by means of a high level of influenza vaccination among nurses and health care professionals in Flemish emergency departments. Responding to professional responsibility does not seem to be an added value [12]. Targeted awareness-raising and winning the confidence of nursing and care professionals will be necessary in order to increase the flu vaccination rate.

\section{Abbreviations}

WHO

World Health Organization

UZA

University Hospital of Antwerp

SPSS

Statistical Package for the Social Sciences

\section{Declarations}

\section{Ethics and Consent to participate}

The research protocol and the questionnaire were approved by the Ethics Committee of the University Hospital of Antwerp (UZA) with the number 17/46520. Participation in the study was non-binding and a digital informed consent was included in the questionnaire. The participants were made anonymous.

After the central ethical committee of the UZA had approved this multicentre study, written contact was made with 56 managing boards of Flemish hospitals that have an approved emergency department. This list of recognised emergency services was obtained via the Federal Public Health Service. The necessary information about the purpose and progress of the research and the digital link to the questionnaire were then provided to the head nurses of the participating hospitals, who in turn invited the respondents via email. The survey covered the period from 14/01/2018 to 28/02/2018.

\section{Competing interests}

The authors declare that they have no competing interests.

\section{Funding}

The authors declare that they not receive any research funding for the article.

\section{Authors contributions}


MS coordinated the study. As principal investigators, MS and HVDV were responsible for all scientific aspects of the work. All authors were involved in the preparation of the research project, the analyses, and the drafting of the manuscript.

HV supervised analysis of the databases and the formulation of results.

PVD and GVH provided scientific support during the various phases of the study. All authors provided feedback and made revisions to the manuscript.

\section{Acknowledgements}

Many thanks to all fellow researchers who participated in this research study: Guido Van Hal (GVH), Herman Vandevijvere (HV), Pierre Van Damme (PVD)

\section{References}

1. WHO. 2018. Definition influenza. World Health Organisation.

2. Galanakis E, Jansen A, Lopalco PL, Giesecke J. Ethics of mandatory vaccination for healthcare workers. Euro Surveill. 2013;18(45):20627.

3. Seizoensgriepvaccinatie van gezondheidswerkers. Wetenschappelijk dossier Vandermeulen C, Boey L, Hoppenbrouwers K. 2017. Seizoensgriepvaccinatie van gezondheidswerkers. Wetenschappelijk dossier.

4. HGR. Vaccinatie tegen seizoensgebonden griep: winterseizoen 2018-2019. Brussel: Federale Overheidsdienst Volksgezondheid, Veiligheid van de Voedselketen en Leefmilieu; 2018.

5. Vandermeulen C, Boey L, Hoppenbrouwers K. 2017. Seizoensgriepvaccinatie van gezondheidswerkers. Handleiding voor ziekenhuizen.

6. Gemeenschap V. 2016. Studie in verband met de motivatie van gezondheidspersoneel over seizoensgriepvaccinatie.

7. Hofmann F, Ferracin C, Marsh G, Dumas R. Influenza vaccination of healthcare workers: a literature review of attitudes and beliefs. Infection. 2006;34(3):142-7.

8. Zhang J, While AE, Norman IJ. Seasonal influenza vaccination knowledge, risk perception, health beliefs and vaccination behaviours of nurses. Epidemiol Infect. 2012;140(9):1569-77.

9. Dominguez A, Godoy P, Castilla J, Maria Mayoral J, Soldevila N, Torner N, Toledo D, Astray J, Tamames S, Garcia-Gutierrez S, Gonzalez-Candelas F, Martin V, Diaz J, Workers C. Knowledge of and attitudes to influenza in unvaccinated primary care physicians and nurses. Hum Vaccin Immunother. 2014;10(8):2378-86. P.W.G.f.t.S.o.I.V.i.P. H.C,. ).

10. Pereira M, Williams S, Restrick L, Cullinan P, Hopkinson NS. Healthcare worker influenza vaccination and sickness absence - an ecological study. Clin Med (Lond). 2017;17(6):484-9.

11. Maridor M, Ruch S, Bangerter A, Emery V. Skepticism toward Emerging Infectious Diseases and Influenza Vaccination Intentions in Nurses. J Health Commun. 2017;22(5):386-94. 
12. Smith S, Sim J, Halcomb E. Australian general practice nurse's knowledge, attitudes and practices regarding influenza vaccination: a cross-sectional survey. J Clin Nurs. 2016;25(17-18):2502-10.

\section{Tables}

Table 1: Research population and demographic data

\begin{tabular}{|c|c|}
\hline \multicolumn{2}{|l|}{ Research population and demographic data } \\
\hline \multicolumn{2}{|r|}{$N=548$} \\
\hline & $\%$ \\
\hline Female & $61 \%$ \\
\hline \multicolumn{2}{|l|}{ Age category } \\
\hline $20-35$ & $37 \%$ \\
\hline $36-50$ & $39 \%$ \\
\hline $51-65$ & $24 \%$ \\
\hline \multicolumn{2}{|l|}{ Educational level } \\
\hline Bachelor of nursing with special professional title 'emergency and intensive' & $85 \%$ \\
\hline Others & $15 \%$ \\
\hline \multicolumn{2}{|l|}{ Jobtime } \\
\hline Full time & $62 \%$ \\
\hline \multicolumn{2}{|c|}{ Mean (SD) } \\
\hline Years active as a health worker & $19 \mathrm{y}(11)$ \\
\hline
\end{tabular}

Table 2: General knowledge of influenza 
According to the WHO, influenza is defined as

Correct definition known

(Infection, lasts about a week, and is characterized by sudden high fever, muscle soreness, headache, severe malaise, non-productive cough, sore throat and rhinitis)

Definition partly known

(Infection, lasts more than two weeks, and is characterized by sudden high fever, muscle soreness, headache, severe malaise, non-productive cough, sore throat and rhinitis)

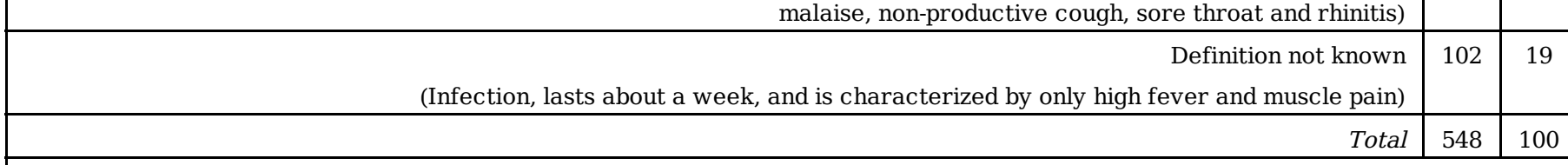

Can I infect others with flu during the incubation period?

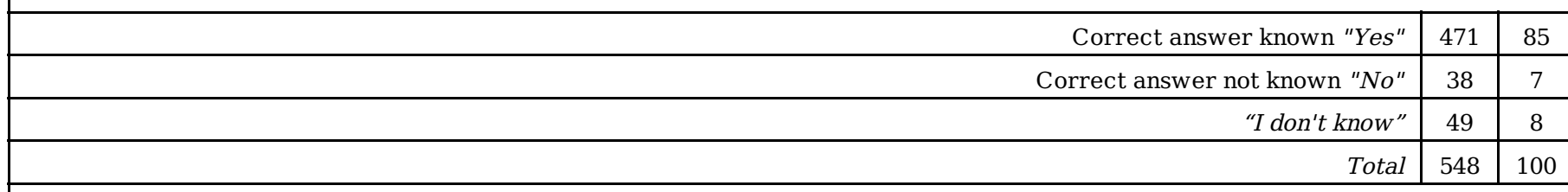

With the symptoms of flu, would I continue to work?

\begin{tabular}{r|c|c|} 
Correct answer not known "Yes" & 65 & 12 \\
\hline Correct answer known "No" & 317 & 58 \\
\hline "Uncertain" & 166 & 30 \\
\hline Total & 548 & 100 \\
\hline
\end{tabular}

\section{4) Can a pregnant employee get the flu vaccine?}

\begin{tabular}{|c|c|c|c|}
\hline & Correct answer not known "Yes" & 412 & 75 \\
\hline & Correct answer known "No" & 48 & 9 \\
\hline & "I don't know" & 88 & 16 \\
\hline
\end{tabular}

